\title{
String Dualities and Empirical Equivalence
}

\author{
Richard Dawid \\ Stockholm University \\ Department of Philosophy \\ 10691 Stockholm, Sweden \\ email: richard.dawid@philosophy.su.se
}

\begin{abstract}
String dualities establish empirical equivalence between theories that often look entirely different with respect to their basic ontology and physical structure. Therefore, they represent a particularly interesting example of empirical equivalence in physics. However, the status of duality relations in string physics differs substantially from the traditional understanding of the role played by empirical equivalence. The paper specifies three important differences and argues that they are related to a subsantially altered view on the underdetermination of theory building.
\end{abstract}




\section{Introduction}

The abundance of duality relations constitutes one of the most important and conspicuous characteristics of string physics. Dual theories are empirically equivalent to each other. They typically share one parameter (like a coupling constant or the radius of a compact dimension) whose value is inverted when switching from a theory to its dual. The same spectrum of values for observables can be found in both theories, but those observables may be interpreted in very different ways in the theory and its dual. Two kinds of string dualities, T-duality and S-duality, play a crucial role in connecting the 5 consistent types of superstring theory, thereby turning the spectrum of possible types of superstring theories into one unique coherent web of 'perspectives' on the same theory. Another very important exemplification of duality is the AdS/CFT correspondence first suggested in Maldacena (1998). This correspondence reaches out beyond string theory proper by relating a string theory on anti-de Sitter spacetime to a conformal field theory on the boundary of that spacetime. Remarkably, this duality relation implies the empirical equivalence of a theory that contains gravity (the string theory) and a theory without gravity (the conformal field theory). In recent years, a lot of work has been invested into attempts to generalize AdS/CFT correspondence to other contexts and maybe eventually move towards a general gauge-gravity duality in physics. One effect of ideas in that direction was the emergence of a new understanding of the relation between string theory and quantum field theory. Both are now increasingly perceived as one overall of closely related concepts which may find a full understanding only when viewed in conjunction.

By constituting the most conspicuous form in which empirical equivalence between theoretical descriptions is found in high energy physics today, duality relations arguably have led to a new view on the phenomenon of empirical equivalence in physics. Identifying empirical equivalence in the form of duality relations has turned into a crucial method for acquiring a deeper and more complete understanding of a fundamental physical theory. The present paper will look at the nature of the change of perspective that takes place when empirical equivalence starts being understood primarily in terms of duality relations. The analysis will rely on a specific historical interpretation of the view on empirical equivalence that was prevalent throughout most of the 20th century (presented in general terms in Section 2). Section 3 will relate that view to Henry Poincaré's conventionalism. The comparison of this 'traditional view' with the picture that emerges in the context of string dualities will demonstrate what is new about the duality based point of view on empirical equivalence (Sections 4 and 5). The corresponding shift will 
then be argued to be exemplary of a general change of the perspective on theory building that has emerged in connection with the evolution of string theory.

\section{Empirical Equivalence in Physics}

The view on empirical equivalence that is characteristic of 20th century physics has been shaped to a considerable degree by the 19th century rise of abstract mathematics, which turned out far richer and more diverse than anyone would have imagined a century earlier and led to a novel view of the role of mathematics in the description of physical phenomena.

At the beginning of the 19th century, mathematics was understood to provide a tool for representing and analyzing the quantitative aspects of observations in a way that relied on human intuitions about the world ${ }^{1}$. It was further taken for granted that micro-physical objects adhered to those basic intuitions as well. Neither the need nor the possibility was acknowledged that mathematics or fundamental physics should or could lead beyond the basic intuitions on which human perception of the everyday world relied. Those intuitions grasped the physical world in terms of solid objects or continua that were exactly located in 3 dimensional flat space and moved along a universal time axis. Mathematics was the tool for representing and parameterizing the dynamics of those objects. I shall call this view the classical intuitive view of physics.

The notion of a 'classical intuitive view' will be helpful in the following for describing the role of abstract mathematics in 19th and 20th century physics. The reasons for using this concept will only become fully clear in Section 4, however, where it will play a crucial role in characterizing the specific status of duality relations in string physics.

Needless to say, already pre-19th century concepts relied on abstractions. Newtonian mechanics, or Aristotelian physics, for that matter, are abstract conceptions which do involve what one may call unintuitive elements. The notion of a "classical intuitive view" is not based on a fictitious point zero of abstraction. By stating the intuitions about physical objects at a given point in time (roughly the early 19th century), it rather introduces a backdrop against which the gradient towards the increasingly unintuitive conceptualizations of later periods can be defined.

The 19th century saw a fundamental shift in the understanding of mathematics. New mathematical conceptualizations like complex analysis and

\footnotetext{
${ }^{1}$ At a philosophical level, this understanding is for example represented by Kant's view on the synthetic a priori character of space and time.
} 
curved and higher dimensional spaces suggested that mathematics was not merely a discipline that could represent human intuitions but was capable of transcending them. The deployment of abstract mathematics made physics reach out beyond the classical intuitive view. New mathematics allowed for representations of observed data that did not rely on the classical intuitions about the spatial structure and ontology of objects. Eventually, physical theories such as special and general relativity or quantum mechanics were developed that reached dramatically beyond the physical posits of classical physics. $^{2}$

The described shift from 'old' to 'new' physics had a substantial effect on the understanding of what was physically possible. 'Old' physics had been based on the understanding that the intuition-based and seemingly unquestionable basic understanding of what the objects of this world were like strictly implied what was a physically possible phenomenon. On that basis, to give one example, Newton could understand his development of scientific theory as a "deduction from the phenomena": for Newton, the data available at the time uniquely implied the true theoretical description and, on its basis, the character of future observations.

Contrary to that view, the new understanding that emerged based on the increasing richness of abstract mathematics and its growing role in physics suggested that physics could come up with an adequate mathematical reconstruction whenever strange deviations from the the classical empirical expectations showed up. Driving this point of view to its radical conclusion, it became plausible to expect that any observational regularity pattern imaginable could be represented by some physical theory based on a sufficiently inventive use of modern mathematics. By accounting for observation patterns that had appeared paradoxical from a classical intuitive view, special relativity and quantum mechanics both appeared as exemplifications of that process. If physical conceptualization based on modern mathematics seemed flexible enough to reproduce any sort of observational regularity, however, there was no reason to assume that just one empirically adequate physical conceptualization was possible. It seemed more natural to expect a system-

\footnotetext{
${ }^{2}$ Note that we are interested in what physicists took to be the physical characteristics of the world. Increased mathematical abstraction such as the deployment of the Hamiltonian or Lagrangian formalism in classical mechanics does not per se constitute a development away from the classical intuitive view as long as the more absract formalism is taken to deal with the same old physical objects. The boundaries between physical characteristics of the world and mathematical formalism become increasingly blurred, however, with the deployment of higher mathematics. Nothing in our analysis hinges on keeping up a strict distinction between the two sides.
} 
atic underdetermination of physical theory building by empirical data. In other words, it seemed plausible to expect that typically several or many empirically equivalent theories could be constructed which all could accurately describe a given observed phenomenon. ${ }^{3}$

At this point, it is important to make some clarificatory remarks on the the relation between the underdetermination of theory building and empirical equivalence. The main focus of this paper lies on the issue of full empirical equivalence of scientific theories. Theories are empirically equivalent if they have identical empirical implications. Underdetermination of theory building in this strong sense means that not even all data that could be collected in principle could fully determine theory building. One might also consider a weaker form of underdetermination, however: underdetermination of theory building under the available data. Underdetermination in this sense has been called "transient" underdetermination by Sklar (1975) and Stanford (2006) and scientific underdetermination in Dawid (2006, 2013). Scientific underdetermination does not imply the existence of fully empirically equivalent theories. It merely implies that there are theories between which one cannot decide based on the available data.

Dawid $(2006,2013)$ makes the point that assessments of scientific underdetermination play a crucial role in motivating trust in string theory in the absence of empirical confirmation. The analysis of empirical equivalence cannot, on its own, provide such reasons. This paper therefore has a different focus. It analyses issues related to the evolution and conceptual understanding of string physics, irrespectively of the question as to whether or not one has good reasons for take the theory to be viable. Still, it is important to point out that the two forms of underdetermination are related. The general conceptual arguments for the existence of empirically equivalent theories that have been stated at the beginning of this section speak also in favour of scientific underdetermination: if the flexibility of theory building can be expected to allow for constructing empirically fully equivalent theories, it may be expected even more strongly that it allows for the construction of theories that are empirically indistinguishable based on the available data. Therefore, important characteristics of the view on empirical equivalence can be nicely illustrated already at the level of scientific underdetermination. We will make use of that similarity at one instance later in the text.

\footnotetext{
${ }^{3}$ This doesn't mean that 'old' physics knew no cases of empirically equivalent theory building. One may just think of the Ptolemaic and the Copernican systems of planetary motion. In the following, however, we shall be mostly interested in the connection between empirical equivalence and the unintuitive developments in modern physics based on advanced mathematics.
} 


\section{Poincaré's Conventionalism}

At the turn of the 20th century, one influential idea provided a conceptual framework for the general considerations discussed in the previous section: Henry Poincaré's conventionalism (1952).

Poincaré's point of departure was the discovery of non-Euclidean space by Gauss, Bolyai and Lobachevsky. In the late 19th century many philosophers took that discovery as a refutation of Kant's claim that (Euclidean) space was synthetic a priori. If more than one kind of space structure was mathematically possible, the decision as to which of those spaces characterized our world seemed to be an empirical question that had to be answered a posteriori by comparing the empirical predictions of physical theories based on each of those space structures with observation. Poincaré agreed with the verdict that the discovery of non-Euclidian geometries established that space was not synthetic a priori. ${ }^{4}$ He denied, however, that this turned the choice of physical space into an empirical question. Poincaré argued that the choice between possible geometries rather amounted to an aspect of theory building that was in principle underdetermined by observation. Each choice of geometry could lead to the same empirical predictions if the physical laws of objects moving and interacting on that space were modified accordingly. In other words, Poincaré believed that the increased range of mathematical conceptualization in the given case did not open up the possibility of representing observational patterns that had no possible scientific description before. Rather, he argued, the new degreees of freedom were fully spent on widening the spectrum of empirically equivalent theories.

The resulting position is called conventionalism. It asserts that, once one fully exploits the freedom of mathematical conceptualization in physical theory building, physical theories become partly conventional. Some elements of those theories can be chosen freely and, once specified, provide a framework for adapting physical concepts to empirical data. The various possible frameworks may not all be equally convenient but all of them provide a basis for constructing an empirically adequate theory. Though Poincaré's claim of conventionalism was most famously applied to the question of space, he suggested that conventionalism was a more general characteristic of physical theory building.

The general view on empirical equivalence that emanates from Poincaré's

\footnotetext{
${ }^{4}$ Poincaré did not fully reject Kant's claim of a synthetic a priori character of mathematics. He did take arithmetic to be synthetic a priori and he tried to identify a synthetic a priori feature on which our concept of space rested, which he called continuity. For a discussion of this point, see e.g. Folina (2006).
} 
conventionalism - I will call it the traditional view on empirical equivalence - closely follows what has been said in general terms at the beginning of this section. Empirically equivalent theories arise due to the high degree of flexibility of physical theory building based on advanced mathematics. There are more degrees of freedom in theory building than there are empirical degrees of freedom the theories have to cover. The excess of theoretical freedom can be exhausted by conventional choices that, in turn, amount to the construction of empirically equivalent theories. ${ }^{5}$

This general view on the idea of empirical equivalence was prevalent in physics throughout most of the 20th century. However, the record of discovering actual physically interesting examples of empirically equivalent theories was modest at best. Poincaré's prime case of the geometry of physical space became far more tricky after the advent of Einstein's theory of general relativity, which has been understood by many - including Einstein himself as a refutation of Poincaré's conventionalist understanding. (For a recent philosophical discussion of that issue, see for example DiSalle (2002).)

Another context where the question of empirical equivalence arose was the case of interpretations of quantum mechanics. However, influential interpretations such as Everettian quantum mechanics or spontaneous collapse models are not fully empirically equivalent to canonical quantum mechanics (Barrett and Byrne 2012, Ghirardi, Rimini and Weber 1986). Even in the case of Bohmian quantum mechanics, the question whether or not it is empirically equivalent to canonical quantum mechanics is a matter of debate (Valentini 1991). ${ }^{6}$

Other examples of empirical equivalence like the one between a canonical formulation of QM in terms of the wave function and a description based on the Feynman path integral formalism, rather than dealing with genuinely different theories, appear as cases of different methods of formulating the same theory.

Remembering the profound conceptual considerations that made the existence of empirically equivalent theories appear as a natural characteristic

\footnotetext{
${ }^{5}$ This paper merely aims at giving a general philosophical reconstruction of the relation between the traditional view on empirical equivalence and Poincaré's conventionalism. It does not aim at doing justice to the nuanced debates on the issue during Poincaré's lifetime and beyond. For discussions of this subject, see Friedman (1996), Zahar (1997) and Ben Menahem (2001, 2012). Other recent discussions of conventionalism are Psillos (2014), and Ivanova (2015).

${ }^{6}$ Interpretations of quantum mechanics that are not fully empirically equivalent to each other do exemplify scientific underdetermination: they are indistinguishable under the available data.
} 
of modern physics from a conventionalist point of view, actual physics until the 1990s had thus produced surprisingly little in terms of actual empirically equivalent theories.

The discovery of a wide spectrum of empirically equivalent theories based on duality relations thus, at first glance, could be read as a late fulfilment of the philosophical considerations of Poincaré and others a century ago. Closer examination reveals a rather different story, however.

\section{Empirical Equivalence and Duality}

Theories with and without gravitons seem empirically profoundly different alternatives. Nevertheless, at least in the case of conformal theories and gravity on AdS space, AdS/CFT duality conjectures that the entire range of observable phenomena that can be accounted for by one of those theories can

also be covered by the other. Graviton theories thus can look like theories without gravity - and theories without gravitons can look like gravitational theories. Fundamental gravitons in this light may be taken to play the role played by curvature in Poincaré's case. They constitute a conceptual element that seems to lead to characteristic empirical signatures. But after close inspection it turns out that those effects can be generated also without including this element in the fundamental theory. A well chosen pure gauge field theory does the job as well. Thus we have two ways of reproducing the same empirical data. There is too much conceptual flexibility in theory building.

On that view, it seems plausible to understand AdS/CFT as an instantiation of empirical equivalence as traditionally understood. Similar lines of reasoning can be found for S- and T- daulity as well. However, closer inspection of the issue reveals three important differences between the case of string dualities and classical exemplifications of the traditional take on empirical equivalence. Those differences shall be discussed the upcoming three subsections.

\subsection{The process of establishing empirical equivalence}

First, we shall consider the emergence of an understanding of empirical equivalence in the two cases.

From a conventionalist point of view, the existence of empirically equivalent theories can be established by new mathematics or new physical mechanisms which enhance the flexibility of fitting theory to data. Once one has understood how the new conceptual tools can be used for theory building and how, on that basis, theories can be tailored to fit empirical data, one 
can reproduce the same data based on various conceptual frameworks. This establishes the empirical equivalence of the corresponding theories.

For example, non-Euclidean geometry introduces a new framework for describing observations of moving objects in space. Once one understands how force laws and background geometry work together for reproducing observations, one can reproduce our observations based on a wide spectrum of background geometries by selecting the force laws accordingly. This results in a spectrum of empirically equivalent theories based on a wide range of background geometries.

It is instructive to point out that the general spirit behind that kind of reasoning is by no means confined to a turn of the 20th century view on physics. A nice though 'imperfect' example of this view on empirical equivalence is the analysis of large extra dimensions in contemporary gauge field theory.

High energy physics provides at least two conspicuous energy scales that lie far beyond the energy levels of the standard model of particle physics. First, the three gauge couplings of the standard model show an energy dependence that makes them meet roughly at one energy scale ${ }^{7}$. This scale, which is about $10^{12}$ times as high as the characteristic scale of the standard model, is called the grand unified scale and provides the basis for introducing fully unified gauge theories at that scale. Second, the gravitational constant, which is many orders of magnitude smaller than the gauge couplings at the standard model scale, grows quadratically with energy and thus assumes values comparable to those of the gauge coupling(s) at the so called Planck scale, which lies about 100 times above the grand unified scale. For a long time, physicists took for granted that the described scale differences denoted actual differences between characteristic energy scales of various types of interaction in 3+1 dimensional spacetime. In the late 1990s it was then suggested that all available empirical data could be reproduced based on the assumption of a higher dimensional spacetime where the extra dimensions were either compactified (Arkani-Hamed, Dimopoulos and Dvali 1998) or showed a particular (warped) geometry (Randall and Sundrum 1999). In those models, some extra dimensions were transgressed by all interactions while others could be transgressed only by gravitation. The altered power laws for interaction at distance in higher dimensional space then implied that both the actual grand unified scale and the actual Planck scale could lie close to observable energy scales. 'Large extra dimension models' play an important role in high energy physics theory building today and we will also encouter them later on in the

\footnotetext{
${ }^{7}$ the meeting of the gauge coupling works much better in a supersymmetric framework.
} 
context of string thery.

Large extra dimension models are not fully empirically equivalent to fourdimensional theories. Once one can test the energy scale of the extra dimensions, they produce characteristic signatures that distinguish them from fourdimensional models. They are empirically equivalent to four-dimensional models at energy scales tested up to this point, however. And within that limited framework, what is going on agrees well with the traditional understanding of empirical equivalence. Once we move beyond our intuition-based prejudice (in this case the prejudice that spacetime is $3+1$ dimensional), new conceptual options open up which provide the basis for new theories that reproduce the same empirical data.

Let us compare this situation with the way dualities typically emerge in string theory. We first look at AdS/CFT duality (Maldacena 1998, Gubser, Klebanov and Polyakov 1998, Witten 1998). The duality relates a string theory on 5 dimensional anti-de Sitter space plus 5 compact dimensions to an $\mathrm{N}=4$ super-conformal theory in 4 dimensions. The latter is a pure gauge field theory with 4 supercharges. This highly symmetric structure enforces a conformal symmetry. Apart from Poincaré invariance, conformal symmetry roughly speaking enforces invariance under transformations that preserve angles but do not preserve lengths. In particular, this means that the theory is scale invariant.

Both theories that are connected by the duality have their conceptual roots in the 1960s and had been developed by the 1980s. String theory began its career as a theory of strong interactions under the name 'dual resonance model' in the late 1960s and was proposed by Scherk and Schwarz as a theory of all interactions in (1974). The first anomaly-free superstring theory found by Green and Schwarz in (1984) established the theory as a promising way to go. It was understood already in the early days of string theory that the physics on the the $1+1$ dimensional string world sheet (the area swept out by a string moving through space) could be described by a 2-dimensional conformal field theory. After the formulation of space-time supersymmetry in 1974 and the following investigations into extended supersymmetries (theories with more than 1 supercharge), it was shown in (Mandelstam 1983) that $\mathrm{N}=4$ supersymmetry (a theory with 4 supercharges) in 4 dimensions was a conformal field theory.

AdS/CFT thus did not reveal the existence of any new theory. The theories related to each other by the duality were long known when the duality was formulated. What the duality did reveal was the fact that for each set of parameter values on one side one could choose the parameter values on the other side in way so that the theories on both sides were empirically equivalent. 
There is a reason why the duality was not formulated in the 1970s or 1980s: Conceptual elements of string theory that were important for seeing the duality were only understood in the 1990s. Those elements played an essential role in motivating the duality conjecture.

To understand this point, we briefly want to rehearse the basic line of reasoning leading up to Maldacena's AdS/CFT duality conjecture in 1998. (See Polchinski's article in this volume.)

Maldacena's derivation of the duality relies on the concept of D3-branes, 3-dimensional objects on which open strings can end. The idea of higher dimensional branes in string physics dates back to the 1980s but a full understanding of D-branes as an essential part of string theory was first achieved by Polchinski in (1995). Another important ingredient in Maldacena's reasoning are black p-branes, extended versions of black holes which arise in a string theoretical context. Those objects were first described by Horowitz and Strominger in (1991). Its connection to D-branes was pointed out by Polchinski in 1995.

Maldacena considers the physics on a stack of coinciding D3-branes, which can be described by a $\mathrm{N}=4$ Super Yang Mills theory, the conformal field theory referred to above.

The effective coupling of this super Yang-Mills theory at energy scale E is

$$
g_{\text {eff }}^{2}(E)=g_{Y M}^{2} N E^{p-3}
$$

Since the gauge fields are massless modes of open strings, we can write the Yang Mills coupling in terms of the string coupling $g_{s}$, which, In the special case $p=3$, leads to

$$
g_{\text {eff }}^{2}=4 \pi g_{s} N
$$

The effective Yang Mills theory will be a good approximation in the perturbative regime, that is if

$$
4 \pi g_{s} N<<1
$$

Seen from "the outside", that is from space beyond the p-dimensional subspace where the Yang Mills theory lives, the stack of coincident Dp-branes constitutes a energetic object that curves spacetime around it. A stack of Dp-branes of sufficient energy density constitutes a black brane, the generalization of a black hole. More specifically, it constitutes an extremal black 
brane where the charge is maximal given its mass (which corrsponds to a situation where all supersymmetries are unbroken). In the 3-brane case, an event horizon of radius $\mathrm{R}$ is created in 5 dimensions of the 6 -dimensional space that is orthogonal to the extended dimensions of the brane. The 6th dimension of that orthogonal space is the radial direction that parametrizes the distance from the brane. $\mathrm{R}$ is

$$
R / l_{s}=g_{e f f}^{-2}
$$

where $l_{s}$ denotes the string length, which means that $R / l_{s}$ is the radius of the event horizon in units of the string length. Space around the black brane forms a peculiar throat geometry where, traveling along the radial dimension towards the brane, the spatial structure of the orthogonal space converges towards a 5 dimensional compactified sphere around the brane with the horizon radius $\mathrm{R}$. (One thus can never reach the brane, unlike in the case of a black hole.) Near the event horizon, the metric of the $3+1$ dimensions parallel to the brane assumes the form

$$
d s^{2}=R^{2} \frac{\eta_{\mu \nu} d x^{\mu} d x^{\nu}+d z^{2}}{z^{2}}
$$

which is the metric of anti-de Sitter space (AdS) with radius $R$ (in Poincare coordinates). The full 10 dimensional metric close to the horizon thus has the form

$$
d s^{2}=R^{2} \frac{\eta_{\mu \nu} d x^{\mu} d x^{\nu}+d z^{2}}{z^{2}}+R^{2} d \Omega_{5}^{2}
$$

which is $\operatorname{AdS}(5) \times S 5$, a 5 dimensional anti de Sitter space with 5 extra dimensions compactified on a 5-sphere. The AdS structure is a good approximation if the radius $\mathrm{R}$ is large compared to the string length $l_{s}$ so that intractable stringy effects from the 5 compact dimensions are suppressed. According to Equations (2) and (4), this is the case if

$$
4 \pi g_{s} N>>1
$$

We thus have found two descriptions of a stack of $\mathrm{N}$ coincident D3-branes which are valid in different limits. The 4-dimensional conformal gauge field theory is valid in the small $g_{s} N$ limit while a supergravity description on 5 -dimensional AdS space is valid in the limit of large $g_{s} N$. 
Maldacena conjectured that the two theories describing the limits of the D3-brane/black p-brane system are dual to each other. He did so by pointing out that a wide range of characteristic properties of the theories match: the number of physical degrees of freedom, the symmetry structure, the spectra of states.

We see that Maldacena makes extensive use of the concept of D-branes and its connection to black branes in order to motivate his conjecture of AdS/CFT duality. He thus relies on conceptual tools that had been developed in the years immediately prior to his work.

The case of $\mathrm{S}$ and T-dualities shows a similar picture.

T-duality relates a string theory with a given radius $\mathrm{R}$ of a compactified dimension to a different string theory with radius $1 / \mathrm{R}$ (in units of the string length $l_{s}$ ). Closed strings moving along the compact dimension are characterized by two discrete numbers: the quantized momentum along the compact dimension (called the Kaluza Klein state) and the times the string is wrapped around the compact dimension (called the winding number). The Kaluza Klein state of the initial theory is equal to the winding number of the T-dual and vice versa. Open strings which move freely through space in the initial theory have no winding number. Therefore, T-duality enforces momentum zero along the compact dimension. This corresponds to the string moving along a hyperplane in the compact dimension that corresponds to a D-brane. By showing that D-branes behave consistently like genuine physical objects, Polchinski established them as a necessary ingredient of string theory.

S-duality relates a string theory with string coupling $g_{s}$ to a string theory with coupling $1 / g_{s}$. First discussions of T- and S-duality date back to the 1980s. Only in the mid 1990s, however, a deeper understanding of the role of dualities revealed that S- and T-dualities in conjunction could connect all known types of string theory (Hull and Townsend 1995, Townsend 1995, Witten 1995, Horava and Witten 1996, Polchinski and Witten 1996).

Soon after the first consistent formulation of a superstring theory by Green and Schwarz, it was understood that one could only build 5 types of superstring theory, which differed from each other in the string boundary conditions, the internal symmetry group, the number of supersymmetries and other properties. The understanding of S- and T-duality that emerged in 1995 implied that all those types of superstring theory were just different empirically equivalent formulations of the same theory.

Unlike in the case of AdS/CFT, the conceptual shifts of the second string revolution in 1995 did lead to the conjecture of a new empirically equivalent (formulation of the) theory. That theory is 11 dimensional M-theory, which still remains very little understood. The core impact of T- and S-duality 
proper, however, is to reveal the coherent web of duality relations between the five types of theories that had already been known since 1985 .

Structurally, the difference between the mindset behind a traditional understanding of empirical equivalence and the mindset behind duality relations thus may be formulated the following way. In both cases, the basic elements that enter the argument are the same. We have

1. a new conceptual tool that allows for kinds of analysis that were not possible before. In our examples, those tools would be:

non-Euclidean geometry in Poincare's gravity example.

extra spatial dimensions in theories of large extra dimensions.

brane physics and related concepts in string dualities.

2. the empirical equivalence claims that are made after the new conceptual tools have been deployed.

But the way those elements are connected is different. In the traditional understanding, the use of the new conceptual tools actually leads to the construction of new theories, which are then understood to be empirically equivalent to theories that had been known before or, if a spectrum of new theories had been developed, empirically equivalent to each other ${ }^{8}$. In the duality cases, the theories were mostly known already from the start. The new tools were mainly deployed for understanding the empirical equivalence of known theories.

One might take this to be a historical contingency that in itself tells little about the character of the involved theories. We should also keep in mind that there is one very important case where a duality relation in string physics indeed hints towards a new previously unknown theory: M-theory. Still, there are conceptual reasons why physicists so often first find the dual theories and only later, based on an additional conceptual step forward, understand that the theories are empirically equivalent. Those reasons do relate to the conceptual situation of string theory and shall be discussed in the next subsection.

\subsection{The role of the classical (intuitive) limit}

The analysis will once again rely on the notion of the classical intuitive view introduced in Section 1. Obviously, string theory is quite remote from the classical intuitive view attributed to early physics. Strings and higher dimensional Calabi Yau spaces clearly played no role in that intuitive world

\footnotetext{
${ }^{8}$ An example of this kind is the understanding that Schroedinger's wave mechanics and Heisenberg's matrix mechanics were empirically equivalent.
} 
view. However, two significant ways can be identified in which it is possible in a string theoretical context to move closer to the classical intuitive view. First, one can look at string theory in the weakly coupled regime and second, one can disregard higher dimensional objects, the so called branes.

Let us first look at the weak coupling limit. Point of departure for doing string theory is to place a string in background space and calculate its dynamics. This is a perturbative approach, perturbing around a background spacetime the curvature of which would be determined entirely by the string dynamics in the full string theory. Perturbative calculations only work, however, if the string coupling is small, so that perturbation theory gives reliable results based on a few orders in the perturbative expansion. Therefore, it is necessary to look at the theory close to a weakly coupled limit.

The weak coupling limit, however, corresponds to the classical limit of the theory. In string theory just like in gauge field theory, an expansion in $g$ also amounts to an expansion in $\mathrm{h}$ since $\mathrm{g}$ and $\mathrm{h}$ always appear to the same order in the terms of perturbative calculations. Sticking to the weakly coupled regime that is calculable based on perturbation theory thus corresponds to sticking to a near-classical regime where quantum fluctuations don't dominate. In other words, looking only at perturbative string theory amounts to looking at the regime of the theory where classical intuitions still work to certain degree.

The second step towards the classical intuitive view consists in disregarding higher-dimensional branes. We have encountered D-branes already in Section 4.1. in the context of T-duality. ${ }^{9}$ A Dp-brane is an infinetely extended object with p spatial dimensions on which open strings can end. Open strings are tied to D-branes while closed strings can move freely away from them. Matter and gauge particles are oscillation modes of open strings, while the graviton is an oscillation mode of a closed string. Therefore, the existence of D-branes (of a dimension different than the dimension of background space) implies that gravitation "feels" a different number of dimensions than the gauge interactions. The classical intuitive view does not know of any dimensions that are not open to all interactions. Opening up this additional degree of freedom thus constitutes a step away from the intuitive view. Disregarding D-branes in turn leads a little closer to the classical intuitive view.

String theory up to the mid 1990s was confined to perturbative string theory since no good method was known to reach out beyond it. And it was - with a few exceptions (see e.g. Bergshoeff, Sezgin and Townsend 1987) disregarding brane physics since the latter seemed overly excentric.

\footnotetext{
${ }^{9}$ There are also other kinds of branes but they won't concern us.
} 
Looking at the theory close to its classical limit and disregarding brane physics makes it impossible to see the duality relations between the various types of string theory, however.

Dual theories in string theory look decidedly different from each other because their classical limits are different physical theories. ${ }^{10}$ This is most conspicuous in the case of S-duality. (see Polchinski, Section 1 in this volume for a more careful development on this argument.) S-duality relates a strongly coupled theory to another theory that is weakly coupled. An analysis that is confined to the weakly coupled /near-classical regime cannot reveal the duality relation since the duality relates one theory in the weakly coupled regime to a strongly coupled dual theory where our intuitions about the dynamics of the theory's "fundamental" objects don't apply. In other words, our near-classical intuition prevents us from having an intuitive grasp of the empirical equivalence between two S-dual theories.

T-duality connects a theory with a large radius $\mathrm{R}$ of a compact dimension to a theory with a small radius. Due to the quantum nature of objects, they cannot be localized in a small compact dimension. The near-classical intuitive picture of localized objects therefore only works in the case of a large compact dimensions R. For that reason, T-duality cannot be grasped based on near-classical intuition. Moreover, T-duality only works due to brane physics. Even if one assumed a superstring theory of a given type that did not contain any D-brane, T-duality would relate that theory to a string theory of a different type that did contain a D-brane. Without D-branes T-duality does not get off the ground.

In the case of AdS/CFT correspondence, only a space with small curvature can be viewed as a background space for propagating near-classical particles. AdS-CFT correspondence relates AdS-space with a small curvature to a strongly coupled field theory, however, that is once again not in a near-classical regime. And once again, AdS/CFT correspondence rely on the introduction of D-branes.

The role of (getting closer to) the classical intuitive view thus is very

\footnotetext{
${ }^{10}$ The classical limit of a quantum theory is a non-trivial issue already in non-relativistic quantum mechanics (see Wallace 2013, Ballentine 1970). The issue whether the classical limit of quantum mechnics is classical mechanics or statistical mechanics is comparable to the case of of different classical limits of string theory in so far as different classical theories can be reached by taking classical limits of the same quantum theory. It is substantially less far-reaching however, because the different classical theories, unlike in the string case, are taken to represent the same actual physical state (since statistical mechanics models the observer's limited knowledge of the system rather than the state's irreducible statistical properties).
} 

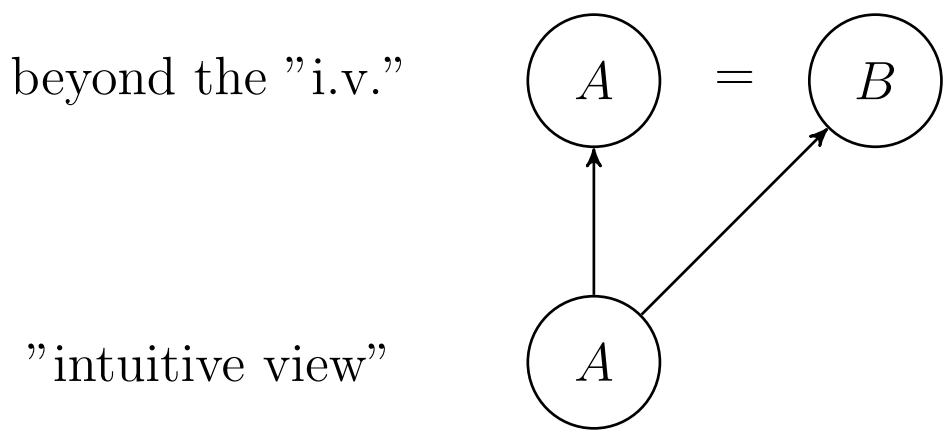

Figure 1: A canonical case of empirical equivalence
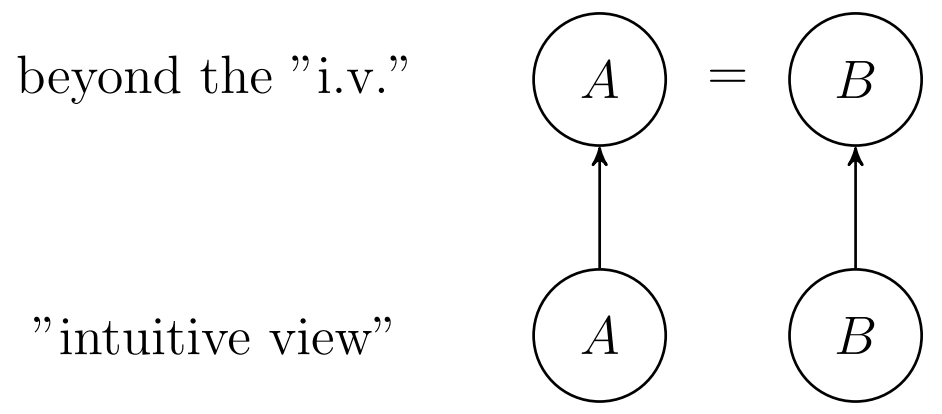

Figure 2: A typical duality case.

different in the case of string dualities than in traditional cases of empirical equivalence. In traditional cases, the detachment of theory building from human intuitions creates new degrees of freedom for theory building. These new degrees of freedom then provide the basis for having more different theories than different possible empirical patterns. This leads to the phenomenon of empirical equivalence. Viewed from the opposite direction, empirically equivalent theories are lost if one removes new conceptual tools. Once non-Euclidean geometries are withdrawn, we are left with one theory in Euclidean space. Once the concept of extra dimensions is withdrawn, we are left with our canonical understanding of the running of gauge couplings in a $3+1$ dimensions background space. The structure of the relation between going beyond the intuitive view and finding empirical equivalence in the traditional case is shown in Figure 1.

In the case of duality, the dual theories mostly have different classical limits. Therefore, considering the classical limits does not make one of the dual theories vanish. It just prevents the understanding of the duality relation. Likewise, it is possible to formulate the theory in the perturbative regime without introducing D-branes. But not accounting for them blocks an understanding of the theory away from that limit. Only once one moves 
away from the regime where our (classical) physical intuition is applicable, and once one takes brane physics into account, the different classical stories merge into one full (quantum) story. The relation between an intuitive point of departure and a non-intuitive analysis thus works the opposite way than in the traditional cases. We have more empirically distinguishable theories in the intuitive (classical) limit than there are empirically distinguishable quantum theories. The structure of the transition from viewing the situation close to the classical limit, which corresponds to the "intuitive view" in the given case, to viewing it after taking into account the full range of unintuitive aspects of the theory is shown in Figure 2.

\subsection{Understanding physics based on empirical equivalence}

The third and arguably conceptually most interesting difference between string dualities and conventional exemplifications of empirical equivalence pertains to the role dualities play in understanding the theory at hand. In the traditional picture, physicists don't need the empirically equivalent theory for attaining a full description of the initial theory. Physicists may get a firmer grip on some physical aspects by approaching them from another conceptual point of view, but only one theory on a subject is needed to fully characterize the physics. For example, non-Euclidean geometry is not needed to fully develop Euclidean physics. Nor are large extra dimensions, qua being an alternative to conventional 4-dimensional theories of grand unification, a means of understanding or completing the latter.

In the case of string dualities, the situation is very different. A good understanding of the entire spectrum of theories is essential for understanding the physical situation. Rather than talking about different theories, it seems more accurate to talk about different dual perspectives on one theory. ${ }^{11}$ The fact that none of the available perspectives is sufficient for fully understanding the theory is rooted in the specific status of the perturbative approach to string theory. As discussed above, perturbative calculations work well close to a classical limit of the theory, in a weak coupling regime. Perturbation theory cannot offer a basis, however, for extracting the dynamics of string theory from the theory's first principles. Since the string coupling itself corresponds to the ground state of an oscillation mode of the string (the dilaton), the specification of a specific (e.g. small) value of the string coupling must itself be based on calculating the theory's dynamics. Therefore, no full calculation

\footnotetext{
${ }^{11}$ Note that the term "perspective" also indicates a difference to an interpretation of the theory. While one may discuss whether one or the other interpretation is correct, different perspectives may be more or less instructive with respect to a given question but one perspective is not more or less correct or true than any other.
} 
of the string coupling can work on a perturbative basis. A full understanding of string theory requires strategies for reaching out beyond the perturbative regime.

Unlike in the case of gauge field theory, however, no full formulation of the equations of motion, no full Lagrangian of string theory, has been found. (One may add that even finding a full formulation of string theory would not mean that the theory's dynamics could be calculated on that basis.) String theorists are therefore confined to making limited inroads into the non-perturbative regime. The most successful strategy to that end has been the use of duality relations. By relating a strongly coupled to a weakly coupled theory, dualities offer possibilities for acquiring information about the strongly coupled regime of a theory that cannot be directly accessed by perturbative methods. Dualities thus play an important role in understanding string theory by extending the knowledge beyond the modest scope of what can be known based on the perturbative analysis of the individual types of string theory.

The five types of superstring theory that are related to each other and to M-theory by duality relations are specified by characterizing the properties of individual strings propagating on a background space. ${ }^{12}$ That is, they are specified within a perturbative framework. Given that the perturbative approach does not allow for a full understanding of the theory, however, the question may be asked: in what sense do the perturbative formulations of different types of string theory represent full-fledged theories at all that can be claimed to be empirically equivalent to each other?

The fact that string theorists feel justifed to make the latter claim is based on the understanding that a perturbative description of string theory, while insufficient for fully understanding the theory, is sufficient for fully specifying it. Specifying a string theory at the perturbative level is taken to determine the characteristics of the full theory: the step from the perturbative level to a full formulation of the theory would not require making any new physical choices or introducing any new physical posits at the fundamental level. ${ }^{13}$

One may cite two general considerations that back this understanding. First, the framework of gauge field theory, which is related to string theory in a number of ways, provides a context where the connection between the full theory and the perturbative regime is better understood and where the

\footnotetext{
${ }^{12}$ For a systematic construction of the five types of string theory, see e.g. Polchinski 1999 .

${ }^{13}$ The situation is very different with respect to the theory's groundstates. It is assumed that a huge number of allowed groundstates exist. Selecting the actual groundstate, is a stochastic quantum process.
} 
perturbative formulation of the theory, while insenitive to a number of nonperturbative effects, stands in a nearly (though not always entirely) unique relation to the full gauge field theory. Second, the strong evidence for duality relations is argued to be difficult to interpret without taking them to be exact (see e.g. Polchinski 2014). Taking duality relations to be exact, however, implies assuming that an exact correspondence relation between dual theories can be established already by specifying the theories within a perturbative framework.

This characteristic of string physics is crucial for allowing statements of empirical equivalence between different types of string theory at all. Only if the individual types of string theory can be specified based on their characterization within a perturbative framework is it possible to develop meaningful claims of duality relations between them. Those duality claims, in turn, strengthen the understanding of the various types of string theory and thereby further contribute to the understanding that they can be specified already at the perturbative level.

The specific role of string dualities with respect to understanding the theory thus is closely related to the peculiar status of the perturbative level of analysis of string theory. On the one hand, the perturbative analysis seems sufficient for uniquely specifying the theory. On the other hand, however, it is too weak for fully understanding and calculating the theory. On this basis, duality relations can be formulated in a meaning full way and, at the same time, can be of substantial use for learning more about string theory.

\section{Conclusion}

The three substantial differences between the traditional view on empirical equivalence and the case of string dualities which I have described in the previous section play out at two levels.

In part, they constitute changes of the role empirical equivalence plays in the scientific process. While in the traditional context cases of empirical equivalence were established by applying new tools to theory building that allowed a wider range of conceptual freedom, in the case of string dualities they are mostly established by using new conceptual tools for establishing the empirical equivalence of theories that were previously taken to empirically distinguishable; and while the existence of empirically equivalent theories previously posed the question which theory should be picked in order to have the most effective (and maybe ontologically convincing) description of the corresponding physics, in the case of string dualities taking all empirically equivalent theories into account is essential for acquiring an understanding of the full theory at all. 
But those pragmatic differences are based on substantial differences with respect to the way the empirically equivalent theories are related to a classical or in some sense more intuitive limit. In the canonical cases, moving to that limit took away degrees of freedom that were necessary for opening up the spectrum of empirically equivalent theories. In the case of string dualities, the different empirically equivalent theories correspond to different classical limits. In the canonical cases, each of the empirically equivalent theories could be fully formulated. In the case of string physics, the dual theories can be established to be empirically equivalent because they can be specified already at the perturbative level. But that level of description does not allow for a full formulation of the theory. In this light, it seems more adequate not to talk about dual theories but about dual perspectives on one theory that needs all those perspectives (and probably more) to be fully characterized. Empirical equivalence thus turns from the phenomenon of multiplicity in theory space to the phenomenon of different perspectives on one theory, which are based on different conceptual starting points that correspond to different classical limits of the theory.

While empirical equivalence seemed to be about the flexibility of conceptualizing observational regularities, in a string theory context it is about the multiplicity of classical limits.

This shift corresponds to a substantial shift with respect to the role of underdetermination of theory building. As discussed in Section 2, the traditional view on empirical equivalence seemed plausible in the face of the increased power and flexibility of physical conceptualization in the late 19th and earlier 20th century. In the face of this increasing strength of conceptual tools that increasingly freed physics from the constraints of intuitions based on everyday observations, it seemed natural to assume that empirically equivalent theories should arise. The further development in physics did not really vindicate those expectations, however. Really convincing examples of empirically equivalent theories were scarce, to say the least. The flexibility of conceptual tools turned out to be compensated by the strong constraints on consistent theory building that emerged throughout the 20th century.

In the context of string theory, the traditional picture becomes fully inadequate. However, a new view on empirical equivalence emerges, where empirical equivalence is understood in terms of the spectrum of classical limits of the full theory. From this point of view, talking about different theories only makes sense once one has introduced elementary objects that can be isolated close to one of the classical limits. What empirical equivalence in the form of duality relations reveals is the fact that the options for building different theories are scarcer than one would imagine when looking at the classical limits of theories. Empirical equivalence thus no more is an 
indicator of the flexibility of theory building but, quite to the contrary, an indicator of the strong constraints on theory building that is not fully visible in the classical limit.

\section{Acknowledgements}

I am grateful to Paolo Aschieri, Radin Dardashti and Casey McCoy and two $\mathrm{u}$ disclosed reviewers for very helpful discussions and comments on a draft version of this paper. This work was partly supported by the DFG grant no DA 1633/2-1.

\section{References}

Arkani-Hamed, N., Dimopoulos, S. and Dvali, G. (1998): "The hierarchy problem and new dimensions at a millimeter", Phys. Lett. B429, 263272 (hep-ph/9803315).

Ballantine, L. E. (1970): "The Statistical Interpretation of Quantum Mechanics", Review of Modern Physics 42, 358-381.

Barrett, J. and P. Byrne (2012): The Everett Interpretation of Quantum Mechanics: Collected Works and Commentary 1955-80, Princeton University Press.

Ben Menahem, Y. (2001): "Convention: Poincaré and Some of His Critics", British Journal for the Philosophy of Science 52, 471-513.

Ben Menahem, Y. (2012): Conventionalism, Cambridge: Cambridge University Press.

Bergshoeff, E., E. Sezgin and P. K. Townsend 1987: "Supermembranes and Eleven-Dimensional Supergravity", Physics Letters B189, 75-78.

Dawid, R. (2006): "Underdetermination and Theory Succession from the Perspective of String Theory", Philosophy of Science 73/3, 298-322.

Dawid, R. (2013): String Theory and the Scientific Method, Cambridge: Cambridge University Press.

DiSalle, R. (2002): "Conventionalism and modern physics: a reassessment", Nous 36:2, 169-200.

Folina, J. (2006): "Poincaré's Circularity Arguments for Mathematical Intuition", The Kantian Legacy in Nineteenth Century Science, M. Friedman and A. Nordmann (eds), Cambridge: MIT Press. 
Friedman, M. (1996): "Poincaré's Conventionalism and the Logical Positivists", Greffe et al. (eds.) 333-344.

Green, M. B. and Schwarz, J. H. (1984): "Anomaly cancellation in supersymmetric $\mathrm{D}=10$ gauge theory and superstring theory", Phys. Lett. B149, 117-122.

Ghirardi, G.C., Rimini, A. and Weber, T. (1986): Unified dynamics for microscopic and macroscopic systems", Physical Review, D34: 470.

Gubser, S. S., I. R. Klebanov and A. M. Polyakov (1998): "Gauge theory correlators from non-critical string theory", Phys. Lett. B428, 105 (hep-th/9802109).

Horava, P. and Witten, E. (1996): "Heterotic and type I string dynamics from eleven dimensions", Nucl. Phys B. 460, 506 (hep-th/9510209).

Horowitz, G.T. and Strominger, A. (1991): "Black strings and P-branes", Nucl.Phys. B 360, 197.

Hull, C. M. and Townsend, P. K. (1995): "Unity of superstring dualities", Nucl. Phys. B438, 109 (hep-th/9410167).

Ivanova, M. (2015): "Conventionalism, Structuralism and Neo-Kantianism in Poincaré's Philosophy of Science", Studies in History and Philosophy of Modern Physics 52, 114-122.

Maldacena, J. (1998): "The large N limits of superconformal field theories and supergravity", Adv. Theor.Math. Phys 2, 231 (arXiv:hepth/9711200).

Mandelstam, S. (1983): "Light cone superspace and the ultraviolet finiteness of the N=4 model", Nucl. Phys. B213, 149-168.

Poincaré (1952): Science and Hypothesis, Dover: Dover Publ. Inc..

Psillos, S. (2014): "Conventions and relations in Poincare's philosophy of Science", philsci-archive: eprint/11362.

Polchinski, J. (1995): "Dirichlet-Branes and Ramond-Ramond Charges", Phys. Rev. Lett. 75, 4724 (arXiv:hep-th/9510017)

Polchinski, J and Witten, E. (1996): "Evidence for heterotic-type I string duality", Nucl. Phys. B460, 525 (hep-th/9510169). 
Polchinski, J. (1999): String Theory, Vol. 2, Cambridge: Cambridge University Press.

Polchinski, J. (2014): "Dualities of Fields and Strings", arXiv 1412.5704.

Randall, R. and R. Sundrum (1999): "A large mass hierarchy from a small extra dimension", Phys. Rev. Lett. 83: 3370-3373 and "An alternative to compactification", Phys. Rev. Lett. 83: 4690-4693.

Scherk, J. and Schwarz, J. H. (1974): "Dual models for non-hadrons", Nucl. Phys. B81, 118-144.

Sklar, L. (1975): "Methodological Conservativism", Philosophical Review 84,384 .

Stanford, K. (2006): Exceeding Our Grasp - Science, History and the Problem of Unconceived Alternatives, Oxford: Oxford University Press.

Townsend, P. K. (1995): "The eleven-dimensional supermembrane revisited", Phys. Lett. B350, 184. (hep-the/9501068).

Valentini, A. (1991): "Signal-locality, uncertainty and the subquantum Htheorem", I and II, Phys. Lett. A, 156, 5-11 and 158, 1-8.

Wallace, D. (2013) "Probability in Physics: Stochastic, Statistical, Quantum", in A. Wilson (ed) "The Arrow of Time in Physics", in A. Bardon and H. Dyke (eds.), A Companion to the Philosophy of Time

Witten, E. (1995): "String theory dynamics in various dimensions", Nucl. Phys B443, 85-126 (arXiv:hep-th/9503124).

Witten, E. (1998): "Anti- de Sitter space and holography", Adv. Theor. Math. Phys. 2, 253 (hep-th/9802150).

Zahar, E. (1997): "Poincaré's Philosophy of Geometry or does Geometric Conventionalism Deserve its Name?", Studies in the History and Philosophy of Modern Physics 28, 183-218. 